\title{
Sequence trap (twin reverse arterial perfusion): a
}

\section{case report}

\section{Introduction}

Mme. Y.D is 21years old and has been married for one year. She has no significant medical or surgical pathological history. It was her first pregnancy. She reports that it is her full term pregnancy.

The patient is referred to our unit for fetal bradycardia.Obstetrical examination finds a longitudinal fetal lie, uterine height at $35 \mathrm{~cm}$, SFH irregular, and uterine contractions were present at the rate of 2 contractions per $10 \mathrm{~min}$. Cervix was $3 \mathrm{~cm}$ dilated at digital vaginal examination, erased at $60 \%$ and its consistency was medium. Presentation was cephalic, water bag was broken and amniotic fluid was clear (Figure 1). The ultrasound flash showed a fetal bradycardia at $80 \mathrm{bpm}$ with eutrophic biometry and a heterogeneous intrauterine image measuring $30 \times 17 \mathrm{~cm}$, with a single homogeneous placental cake. Patient had a cesarean section for fetal bradycardia, with the extraction of the first male twin, birth weight $3100 \mathrm{~g}$, Apgar 9/10, then the extraction of a second acardiac twin consisting of 3 segments: upper (probably The head), body and a lower part (one limb). Delivery was directed. Venous and arterial anastomoses were found at the placental examination.
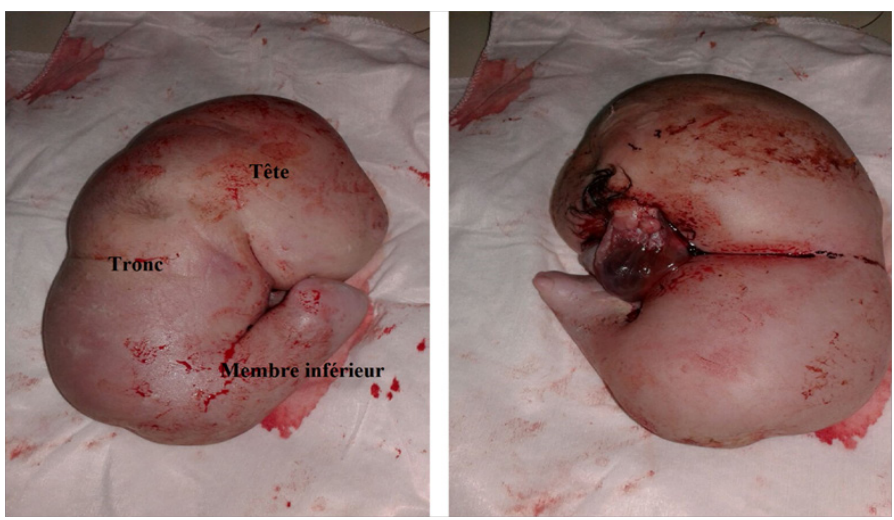

Volume II Issue 6 - 2018

\author{
Ahmed Guennoun, Yousra Krimou \\ Department of Obstetrics and Genecology I, Hassan II \\ University Hospital, Morocco
}

Correspondence: Ahmed Guennoun, Department of Obstetrics and Genecology I, Hassan II University Hospital, Morocco,Email gahmedn@hotmail.com

Received: April 02, 2018| Published: November 19, 2018

Keywords: obstetrical examination, digital vaginal examination, amniotic fluid, placental cake, intrauterine image

\section{Acknowledgements}

None.

\section{Conflict of interest}

Authors declare that there is no conflict of interest.

Figure I Twin reverse arterial perfusion. 